\title{
Valoración de inversiones en proyectos no convencionales - tasa interna de retorno versus tasa interna de retorno modificada
}

\section{Valuation of investments in unconventional projects - internal rate of return versus modified internal rate of return}

Diana Taco

Tablet School-Escuela de Ciencias y Centro de Investigación Científica, Ecuador Marcos Gutiérrez

Tablet School-Escuela de Ciencias y Centro de Investigación Científica, Ecuador Autor para correspondencia: dianataco@tablet-school.com

Fecha de recepción: 23 de junio de 2018 - Fecha de aceptación: 15 de septiembre de 2018

Resumen: Siendo la industria manufacturera uno de los sectores que ha tenido mayor crecimiento económico en el Ecuador se considera necesario comprender la dinámica del negocio, empleando técnicas y herramientas que faciliten el proceso en la toma de decisiones organizacionales. Por medio de la elaboración de flujos de fondos se pretende determinar de forma cuantitativa el saldo final de ingresos y egresos a ello complementándolo con la obtención de índices de rentabilidad tales como: el valor actual neto (VAN), tasa interna de retorno (TIR), período de recuperación y el índice de rentabilidad, se podrá determinar la viabilidad económica en una inversión, sin embargo, existe otra medida de rentabilidad que es de gran importancia y que se aplica en proyectos no convencionales que requieren otro tipo de evaluación, tal es el caso de la Tasa Interna de Retorno Modificada (TIRM). En la presente investigación se desarrolla un método de evaluación de proyectos de inversión diferente a los tradicionales. Se encuentra enfocada al cálculo, análisis y comparación de la Tasa interna de retorno versus la Tasa interna de retorno modificada a partir de la construcción del flujo de fondos proyectado, considerando que, la tasa interna de retorno modificada fue diseñada con la finalidad de superar las inconsistencias de la TIR clásica. Para este análisis se tomó como referencia dos proyectos de una empresa del sector manufacturero del país con periodos de evaluación entre el 2015 y 2016; para posteriormente elaborar la proyección del flujo de fondos con un horizonte de tiempo diferente para cada uno, de 10 y 5 años. Por lo general cuando se realiza un flujo de fondos de un proyecto, y cuando se calcula la TIR se asume que todos los valores del flujo son positivos. Sin embargo, no en todos los proyectos los resultados del flujo son positivos, es decir, los valores pueden ser positivos y negativos y como consecuencia puede surgir varias TIR o ninguna.

Palabras claves: proyectos de inversión; evaluación de proyectos de inversión; análisis de las inversiones públicas y privadas

Abstract: Being the manufacturing industry one of the sectors that has had greater economic growth in Ecuador it is considered necessary to understand the business dynamics, using techniques and tools that facilitate the process in making organizational decisions. Through the elaboration of cash flows, it is intended to quantitatively determine the final balance of income 
and expenses, complementing it with obtaining profitability indices such as: the net present value (NPV), internal rate of return (IRR)., the recovery period and the profitability index, the economic viability of an investment can be determined, however, there is another measure of profitability that is of great importance and that is applied in unconventional projects that require another type of evaluation, such as the case of the Internal Rate of Modified Return (TIRM). In the present investigation a method of evaluation of investment projects different from the traditional ones is developed. It is focused on the calculation, analysis and comparison of the internal rate of return versus the internal rate of return modified from the construction of the projected cash flow, considering that the modified internal rate of return was designed to overcome the inconsistencies of the classic IRR. For this analysis, two projects from a company in the country's manufacturing sector with evaluation periods between 2015 and 2016 were taken as reference; to later elaborate the projection of the flow of funds with a different time horizon for each one, of 10 and 5 years. Usually when a cash flow of a project is made, and when calculating the IRR it is assumed that all the values of the flow are positive. However, the results of the flow are not positive in all the projects, that is, the values can be positive and negative and as a consequence, several IRRs can arise or none at all.

Key words: investment projects; evaluation of investment projects; analysis of public and private investments

\section{Introducción}

Considerar a la TIR como medida de rentabilidad podría ocasionar que la toma de decisiones en una inversión sea incorrecta; de hecho, en base a estudios realizados indican que entre el VAN y el TIR la mejor medida de rendimiento es el VAN. Las inconsistencias de la TIR clásica se originan cuando en un flujo de fondos a parte de la inversión inicial pueden presentar más de un flujo negativo, por lo que se podría obtener varias TIR o ninguna. Al tratarse de proyectos con objetivos y de naturaleza diferente el análisis de los resultados requiere un estudio más profundo, es decir, en base a los cálculos obtenidos de la aplicación de los indicadores financieros es necesario que la interpretación de resultados considere detalles tale como: el monto de la inversión, si los flujos de retorno del dinero fueron generados al inicio o al final del proyecto (el valor del dinero en el tiempo), si el proyecto está financiado o si la empresa utiliza recursos propios. Las herramientas financieras no toman decisiones por sí solas, requieren de la experiencia y del sano juicio del tomador de decisiones. En esta investigación se ejecutó la proyección de dos flujos de fondos con un período de análisis de 10 años y otro de 5 años y además del cálculo de los indicadores tradicionales complementar esta investigación con la aplicación de la tasa interna de retorno modificada.

Algunos autores mencionan que la tasa interna de retorno clásica no debería usarse para ordenar alternativas de inversión, este juicio se inicia con un análisis realizado por Jack Hirshleifer (1958), y más detalladamente por Gerald Fleischer y James Mao (1966) y se mantiene hasta el presente en todos los casos en que se considera que la medida verdadera de una inversión es el valor actual neto, y, que la TIR es válida solo cuando es compatible con ése. [1] Por ello Steven Lin (1976) plantea lo que denomina TIR modificada de un flujo de fondos con reinversión y posibles importes negativos intermedios [1]; considerando además que la TIR normal asume méritos sólo del proyecto, en contraste a esto la TIRM toma en cuenta la tasa de financiamiento y la del costo de capital o denominada también tasa de rentabilidad esperada. En base a estos antecedentes es importante efectuar los cálculos de estos dos indicadores 
conjuntamente con el VAN, a fin de determinar las diferencias encontradas en la aplicación de los mismos y describir qué medida debería considerarse en la toma de decisiones en términos de rentabilidad.

Cuando se evalúa proyectos de inversión se debe considerar que existen proyectos con diferentes características, por lo tanto, la aplicación de modelos financieros es diferente para cada caso y es de vital importancia que los analistas sepan identificar ciertas variables a fin de realizar un análisis coherente de los mismos. Cuando nos referimos a proyectos no convencionales se hace referencia a aquellos proyectos de inversiones no simples, inversiones que además del pago inicial generan, en algún otro año, una cantidad negativa. [2]

En proyectos de este tipo el cálculo del VAN y la TIR no son suficientes para la toma de decisiones, es necesario aplicar la TIRM como una alternativa de solución para las limitaciones de la TIR clásica, debido a que:

1. La TIR es definida también como la tasa interna o intrínseca al proyecto y no depende de otra cosa que no sean los flujos de efectivo del proyecto.

2. La TIR puede ser un criterio ambiguo, ya que no distingue entre la tasa resultante del flujo de fondos de un proyecto y de un préstamo. [1]

3. La TIR hace caso omiso de los problemas de escala. [3]

4. Depende de los flujos de efectivo del proyecto

5. No depende de la tasa de rentabilidad esperada por el inversor (costo de oportunidad)

6. Solucionar el problema de que teóricamente habrá tantas TIR como cambios de signos haya en el flujo de fondos

7. Conocer si los resultados obtenidos nos llevan a la misma decisión de inversión por medio de la aplicación del VAN.

De acuerdo a la descripción presentada se utiliza la TIRM porque, es la tasa de descuento a la cual el valor presente de los flujos de salida de efectivo de un proyecto es igual al valor presente de su valor terminal, donde este último se obtiene como la suma de los valores futuros de los flujos de entrada de efectivo compuestos a la tasa de rendimiento requerida por la empresa; por su parte, el valor presente de los flujos de salida de efectivo se encuentra aplicando la misma tasa de rendimiento requerida. [4] Y el criterio de decisión es que un proyecto es aceptable si la TIRM obtenida es mayor a la tasa de rentabilidad esperada por el inversionista.

Para la aplicación del método se evaluó dos proyectos excluyentes por medio de la aplicación de la Tasa Interna de Retorno modificada (TIRM); exaltando los beneficios de este índice versus la Tasa Interna de Retorno clásica.

\section{Proceso de cálculo}

El objeto de estudio son dos proyectos mutuamente excluyentes de una empresa de manufactura del Ecuador dedicada a la producción y comercialización de productos lácteos en los períodos 2015-2016. Entendiéndose como mutuamente excluyentes a aquellos proyectos que están siendo evaluados para determinar la conveniencia de elegir uno de ellos o a su vez rechazar los dos, dependiendo de los resultados obtenidos de la aplicación de los indicadores de 
rentabilidad VAN, TIR y TIRM. Se analizó los estados financieros de la organización de este sector [5], [6], [7] para luego proyectar los flujos de fondos para un período de 10 y 5 años.

Generalmente en la proyección de flujos de fondos se lo realiza asumiendo que el proyecto genera flujos positivos, en varios casos se presentan proyectos que no generan este tipo de flujos. Puede haber varios cambios en los signos y es allí donde la aplicación de la TIR clásica puede tomar varios valores, por consiguiente, este indicador no representa un criterio financiero confiable a efectos de medir la rentabilidad de un proyecto. Considerar las tasas de interés implícitas solamente en el proyecto también genera una discrepancia respecto de la realidad, un proyecto se encuentra expuesto a factores externos que podrían perjudicar la consecución del mismo. Para resolver este inconveniente al considerar la TIRM como un método adicional a los indicadores VAN y TIR complementa de mejor manera los criterios de evaluación en una inversión, ya que, en esta medida se considera dos tipos de tasas que son fundamentales, tales como: la tasa de rentabilidad esperada conocida también como el costo de oportunidad y la tasa de financiamiento del proyecto.

\section{Ejecución de la proyección}

Siendo el objeto de estudio dos proyectos tomados como referencia, se procedió a estimar los flujos de efectivo para cada uno. El proyecto que corresponde al horizonte de evaluación de 10 años que comprende desde el 2017 al 2026; es un proyecto de elaboración de helados que se financia con un crédito bancario (en adelante denominaremos proyecto 1). El proyecto de 5 años de evaluación corresponde al período de tiempo desde el 2017 al 2021; es un emprendimiento de elaboración de yogures el cual se financia con recursos propios (en adelante denominaremos proyecto 2). Para la proyección de cada uno se tomó como referencia información recopilada de ofertas públicas presentados por la Bolsa de Valores de Quito en el período 2015-2016. [8]

Los criterios de evaluación utilizados fueron el valor actual neto, la tasa interna de retorno y la tasa interna de retorno modificada. Los indicadores tales como el período de recuperación y el índice de rentabilidad no fueron aplicados en esta investigación porque cuando se evalúa proyectos mutuamente excluyentes los resultados de éstos no son consistentes.

En el primer proyecto, el valor actual neto (VAN) y la tasa interna de retorno (TIR) cuyas fórmulas se presentan en las ecuaciones 1 y 2 respectivamente, se calculan en base a los valores obtenidos de los ingresos y egresos con los que se elabora el flujo de fondos. Para la obtención de la tasa de descuento se utilizó la técnica del CAPM (Capital Asset Pricing Model) que es un modelo de valuación de activos de capital y que es utilizada como herramienta para la determinación del rendimiento mínimo esperado a los efectos de tomar la decisión de aceptar o no el proyecto, la misma que será empleada en los dos proyectos por tratarse del mismo sector de análisis (comparables). Su fórmula es la siguiente:

Dónde:

$$
R_{s}=R_{F}+\beta *\left(R_{M}-R_{F}\right)
$$

$$
\begin{gathered}
\mathrm{R}_{\mathrm{s}}=\text { tasa de rentabilidad esperada } \\
\mathrm{R}_{\mathrm{F}}=\text { tasa libre de riesgo }
\end{gathered}
$$


$\beta=$ beta de la compañía

$\mathrm{R}_{\mathrm{M}}-\mathrm{R}_{\mathrm{F}}=$ diferencia entre el rendimiento esperado sobre el portafolio del mercado y la tasa libre de riesgo.

Tabla 1. Datos para el cálculo del CAPM.

$$
\begin{array}{ll}
\hline \mathbf{R}_{\mathbf{F}} & \mathbf{2 . 4 5 \%} \\
\hline \boldsymbol{\beta} & 1.05 \% \\
\hline \mathbf{R}_{\mathrm{M}} & 6.47 \%
\end{array}
$$

Fuente: Elaboración propia.

Aplicando la fórmula (1):

$$
\begin{aligned}
& \mathrm{R}_{\mathrm{s}}=2,45 \%+\% 1,05 \% \cdot(6,47 \%-2,45 \%) \\
& \mathrm{R}_{\mathrm{S}}=6,67 \%
\end{aligned}
$$

\section{Resultados}

Los resultados obtenidos con los indicadores financieros y con las fórmulas abajo descritas, se detallan a continuación:

Donde:

$$
V A N=\sum_{t=1}^{n} \frac{V_{t}}{(1+k)^{t}}-I_{0}
$$

$\mathrm{V}_{\mathrm{t}}$ : representa los flujos de caja en cada periodo $\mathrm{t}$.

$\mathrm{I}_{0}$ : es el valor del desembolso inicial de la inversión.

n: es el número de períodos considerado.

t: es el tiempo

k: es el tipo de interés.

$$
T I R=\frac{-I+\sum_{i=1}^{n} F_{i}}{\sum_{i=1}^{n} i * F_{i}}=0
$$

Donde:

$F_{i}$ : es el flujo de caja en el período t.

n: es el número de períodos.

I: es el valor de la inversión inicial

Tabla 2. Resultados de cálculo.

\begin{tabular}{lc}
\hline VAN & $\$ \mathbf{2 6 5 . 1 7 2 , 9 6}$ \\
\hline TIR & $10 \%$
\end{tabular}

Resultados del VAN y el TIR del Proyecto 1.

Para el cálculo de la tasa interna de retorno modificada (TIRM) se ha diferenciado dos tipos de tasas: la tasa de financiamiento que es la tasa de interés que se paga por el dinero solicitado al banco, en base a la siguiente fórmula se obtuvo el siguiente resultado: 
Donde:

$$
\operatorname{TIRM}=\left[\frac{\sum_{t=1}^{n} C F_{t} *(1+i)^{n-t}}{I}\right]^{\frac{1}{n}}-1
$$

$$
\begin{aligned}
& \mathrm{C}_{\mathrm{F}} \text { : Flujo de Caja } \\
& \mathrm{I} \text { : Inversión } \\
& \mathrm{i}: \text { tasa de descuento } \\
& \mathrm{n}: \text { vida útil } \\
& \mathrm{t}: \text { periodo de tiempo }
\end{aligned}
$$

Tabla 3. Resultados de cálculo. TIRM 9\%

Resultados de la TIRM del Proyecto 1.

Para el segundo proyecto los resultados obtenidos de la aplicación de las fórmulas presentadas precedentemente fueron:

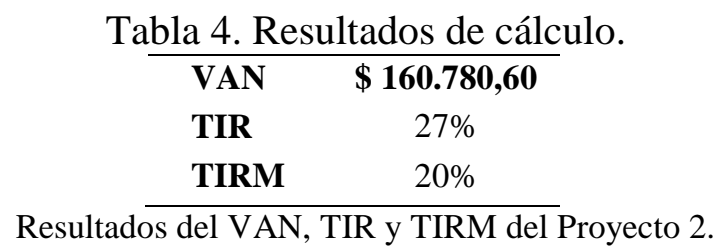

En base a la obtención de los valores del VAN la TIR y la TIRM, se puede efectuar un análisis comparativo entre los resultados calculados con la finalidad de seleccionar el proyecto que genere mayor rentabilidad.

\section{Discusión}

El criterio de selección que aplica la tasa interna de retorno clásica radica básicamente en comparar esta tasa con la del coste de oportunidad del capital o tasa de descuento que en este caso es de 6,67\%; donde el proyecto será aceptado si la TIR es mayor que esta tasa y por lo contrario rechazar el proyecto si la TIR es menor que el coste de oportunidad. Bajo esta regla de selección de proyectos en base a los resultados obtenidos escogeríamos el proyecto de la elaboración de yogures puesto que la TIR es del $27 \%$ con respecto del proyecto de elaboración de helados cuyo resultado es del 10\%. Los resultados obtenidos del cálculo de la TIR indican que ambos proyectos son viables económicamente ya que los dos superan la tasa de rentabilidad esperada (tasa de descuento). Por otro lado, si analizamos los resultados obtenidos del valor actual neto que fueron de $\$ 265,172.96$ para el proyecto 1 y de $\$ 160,780.60$ para el proyecto 2 , vemos que el proyecto 1 es más rentable, por lo que nos encontramos frente al problema de selección entre proyectos mutuamente excluyentes. Tanto el VAN como la TIR deberían llevarnos a la misma decisión, sin embargo, vemos que en este caso los criterios entre ambos son totalmente opuesta. Entonces la interrogante es: ¿Qué proyecto es el mejor? De acuerdo a estudios realizados indican que el VAN es mejor, porque selecciona el proyecto que agrega más valor al patrimonio de los accionistas [9]. 
En primera instancia y bajo este análisis podemos verificar que la TIR hace caso omiso de los problemas de escala, ya que, si bien el proyecto 2 tiene un porcentaje más alto, no considera que el monto de la inversión inicial del proyecto es mucho más pequeño que la del proyecto 1 de allí la primera diferencia en los valores obtenidos en el VAN.

En los proyectos objeto de estudio se presentan varias alternativas en la selección de uno de ellos, el tamaño y el horizonte de tiempo son diferentes, cada uno tiene un objetivo económico diferente por lo que esto nos lleva a efectuar otro análisis y es que tan bueno sería para la empresa que un proyecto genere flujos de fondos en los primeros años y el otro en los últimos años del período de evaluación. Para resolver esta duda se realizó un análisis de las tasas utilizadas en el cálculo del VAN, la TIR y la TIRM. Financieramente evaluando proyectos de inversión los indicadores que presentan mayor aceptabilidad en sus cálculos es el VAN y la TIRM ya que como se mencionó anteriormente los dos consideran para su cálculo la tasa de rentabilidad esperada por el inversor mientras que la TIR solamente toma en cuenta la tasa generada por el proyecto. Siguiendo este análisis podemos mencionar que entre la TIR normal y la TIRM la segunda es la mejor opción en criterios de inversión, a esto complementándolo con el análisis del VAN. Como muestra en la tabla 3 vemos que la TIRM para el proyecto 1 es del 9\%, mayor que la tasa de oportunidad, y el VAN es mucho mayor que el proyecto 2 por lo que se confirma que el proyecto es rentable. En la tabla 4 se describe el valor de la TIRM del proyecto que fue del $20 \%$, pero, en términos de decisión económica el proyecto no es rentable porque el VAN es mucho menor al proyecto de la elaboración de helados (proyecto 1).

\section{Conclusiones}

Para evaluar proyectos mutuamente excluyentes con diferentes horizontes de tiempo y diferente tamaño, es importante conocer las propiedades de cada indicador, identificar las herramientas que permitan tomar las decisiones más acertadas al momento de ejecutar un proyecto nuevo. Y más allá de eso por medio de esta metodología se puede elegir el proyecto más rentable entre varios que pueden ser planteados.

Es importante evaluar cada uno de los flujos de fondos porque puede originarse errores de cálculos, la extensión de la TIR normal se origina por las limitaciones en su cálculo en proyectos no convencionales en los cuales se puede presentar cambios frecuentes de signos.

Se debe considerar que las tasas aplicadas en los cálculos de la TIR y TIRM son diferentes, se debe evaluar las variables de cada una para conocer la medida más acertada para la toma de decisiones.

Para complementar el análisis de las diferencias entre la TIR y TIRM y a fin de tomar el mejor criterio de selección entre varios proyectos se debe efectuar este estudio con las ventajas de utilizar el VAN en proyectos no convencionales.

\section{Bibliografía}

Aire, T. (2014). Conducción Estratégica para la Evaluación de Proyectos de Inversión. Buenos Aires: EDICON. 
Besley, S., \& Brigham, E. (2013). Finanzas Corporativas. México: Cengage Learning. Jeffrey, R. S. (2012). Finanzas Corporativas. México: McGraw-Hill Interamericana Editories S.A. De C.V.

Bolsa de Valores de Quito (2018). Prospectos. Recuperado de: https://www.bolsadequito.com/index.php/mercados-bursatiles/emisores/prospectos. Acceso en, Julio 2018.

Edmundo, P. (2008). Formulación y Evaluación de Proyectos de Inversión.

Ehrhardt, M., \& Brigham, E. (2007). Finanzas Corporativas. México: Cengage Leaming Editores S.A.

Ehrhardt, M., \& Brigham, E. (2007). Finanzas Corporativas. México: Cengage Leaming Editores S.A.

González, N. (2014). Inversiones Puras, Mixtas, Simples y no Simples. Administración Financiera. Recuperado de: https://finanzas-v-sem-cont.webnode.es/news/a3-1-a-inversiones-purasmixtas-simpes-y-no-simples-/. Acceso en, Julio 2018.

Jaffe, Ross, \& Westerfield, 2012, Jaffe, J. F., Ross, S. A., \& Westerfield, R. W. (2012). Finanzas Corporativas. México: McGraw Hill.

Jover, J. (2012). Utilidad del VAN y la TIR en los flujos de caja. Consultoría Financiera. Obtenido de: http://jeronimojover.blogspot.com/2012/09/utilidad-del-van-y-la-tir-en-los-flujos.html

López, G. (2006). Cálculo Financiero Aplicado. Buenos Aires: La Ley.

Prospecto de Oferta Pública (2012). Emisión de obligaciones Heladosa S.A. Recuperado de: https://www.bolsadequito.com/uploads/mercados/emisores/prospectos/E-

H/HELADOSA/OBLIGACIONES/HELADOSA\%20OBL\%202012.pdf. Acceso en, Julio 2018.

Prospecto de Oferta Pública (2017). Recuperado de: https://www.bolsadequito.com/uploads/mercados/emisores/prospectos/AB/ALIMEC/OBLIGACIONES/ALIMEC\%20OBL\%202017.pdf. Acceso en, Julio 2018.

Sapag Chain, N., \& Sapag Chain, R. (Quinta edición 2008). Preparación y evaluación de proyectos. Colombia: McGraw Hill.

Sapag, C. N. (2011). Proyectos de Inversión. Formulación y Evaluación. Chile: Pearson Educación de Chile S.A.

Tapia, G. (2013). Informe Especial Tasa Interna de Retorno Modificada. Revista de Asesoría especializada. Recuperado de: https://es.scribd.com/document/216078451/Tasa-Internade-Retorno-Modificada. Acceso en, Julio 2018. 
Tasa de interés. (2012)¿Cómo calcular la TIR Modificada? Obtenido de: http://tiie.com.mx/comocalcular-tir-modificada/

Tonicorp (2011). Recuperado de: https://www.bolsadequito.com/uploads/mercados/emisores/prospectos/QZ/TONICORP/ACCIONES/TONICORP\%20ACC\%202011.pdf. Acceso en, Julio 2018. 